International Journal of Pure and Applied Mathematics

Volume $100 \quad$ No. 4 2015, 479-483

ISSN: 1311-8080 (printed version); ISSN: 1314-3395 (on-line version)

url: http://www.ijpam.eu

doi: http://dx.doi.org/10.12732/ijpam.v100i4.7

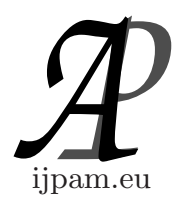

\title{
DYNAMICS OF HYPERBOLIC WEIGHTED COMPOSITION OPERATORS
}

\author{
Bahmann Yousefi ${ }^{1}$, Fariba Ershad ${ }^{2} \S$ \\ ${ }^{1,2}$ Department of Mathematics \\ Payame Noor University \\ P.O. Box 19395-3697, Tehran IRAN
}

\begin{abstract}
In the present paper we investigate conditions under which a hyperbolic self-map of the open unit disk induces a hypercyclic weighted composition operator in the space of holomorphic functions on the unit ball in $\mathbf{C}^{N}$.
\end{abstract}

AMS Subject Classification: 47B33, 47B38

Key Words: weighted composition operator, hypercyclic operator, DenjoyWolff point, hyperbolic map

\section{Introduction}

For $z=\left(z_{1}, \ldots, z_{N}\right)$ and $w=\left(w_{1}, \ldots, w_{N}\right)$ in $\mathbb{C}^{N}$, write $<z, w>$ for the Euclidean inner product $\sum_{j=1}^{N} z_{j} \bar{w}_{j}$ and let $|z|=<z, z>^{1 / 2}$. With this notation, the unit ball in $\mathbf{C}^{N}$ is the set $B_{N}=\left\{z \in \mathbb{C}^{N}:|z|<1\right\}$ and the unit sphere in $\mathbf{C}^{N}$ is the set $S_{N}=\left\{z \in \mathbf{C}^{N}:|z|=1\right\}$, analogously to the unit disc and circle for $N=1$. The space $H\left(B_{N}\right)$, is the set of all holomorphic fuctions on $B_{N}$, can be made into a F-space by a complete metric for which a sequence $\left\{f_{n}\right\}$ in $H\left(B_{N}\right)$ converges to $f \in H\left(B_{N}\right)$ if and only if $f_{n} \longrightarrow f$ uniformly on every compact subsets of $B_{N}$. Each $\varphi \in H\left(B_{N}\right)$ and holomorphic self-map $\psi$ of $B_{N}$ induces a linear weighted composition operator $C_{\varphi, \psi}: H\left(B_{N}\right) \longrightarrow H\left(B_{N}\right)$ de-

Received: December 24, 2014

(C) 2015 Academic Publications, Ltd.

${ }^{\S}$ Correspondence author url: www.acadpubl.eu 
fined by $C_{\varphi, \psi}(f)(z)=\varphi(z) f(\psi(z))$ for every $f \in H\left(B_{N}\right)$ and $z \in B_{N}$. Indeed, $C_{\varphi, \psi}=M_{\varphi} C_{\psi}$ where $M_{\varphi}$ denotes the operator of multiplication by $\varphi$ and $C_{\psi}$ is a composition operator by means of the definition $C_{\psi}(f)=f \circ \psi$ for every $f \in H\left(B_{N}\right)$.

A bounded linear operator $T$ on a F-space $X$ is said to be hypercyclic if there exists a vector $x \in X$ for which the orbit $\operatorname{Orb}(T, x)=\left\{T^{n} x: n \in \mathbb{N}\right\}$ is dense in $X$ and in this case we refer to $x$ as a hypercyclic vector for $T$.

The holomorphic self maps of $B_{N}$ are divided into classes of elliptic and non-elliptic. The elliptic type is an automorphism and has a fixed point in $B_{N}$. It is well known that this map is conjugate to a rotation.

For simplicity, throughout this paper we use the notation $" \stackrel{k}{\rightarrow}$ " for indicating uniform convergence on compact subsets of $B_{N}$. Also, by $\psi_{n}$ we denote the nth iterate of $\psi$. To state the main result of the paper, we need the following theorems from [3].

Theorem 1.1. (Denjoy-Wollf Iteration in $B_{N}$ ) Suppose $\psi$ is a holomorphic self-map of the open unit ball $B_{N}$ without interior fixed point. Then there is a point $w \in \partial B_{N}$ such that $\psi_{n} \stackrel{k}{\rightarrow} w$ and $0<d(w) \leq 1$ where

$$
d(w)=\lim _{|z| \rightarrow 1^{-}} \inf \frac{1-|\psi(z)|^{2}}{1-|z|^{2}} .
$$

The boundary point $w$ is called the Denjoy-Wollf point of $\psi$.

Theorem 1.2. (Julia's Lemma in $B_{N}$ ) Let $\psi$ be an analytic map of the unit ball into itself with Denjoy-Wolff point $w \in \partial B_{N}$. Then for every $z \in B_{N}$,

$$
\frac{|1-<\psi(z), w>|^{2}}{1-|\psi(z)|^{2}} \leq d(w) \frac{|1-<z, w>|^{2}}{1-|z|^{2}} .
$$

Recall that a holomorphic self-map $\psi$ of $B_{N}$ is called elliptic if $\psi$ has a fixed point in $B_{N}$. Also, if $\psi$ has no interior fixed point, then it is called hyperbolic whenever $d(w)<1$, and is called parabolic if $d(w)=1$.

For simplicity, we call a weighted composition operator $C_{\varphi, \psi}$, a hyperbolic weighted composition operator whenever the compositional symbol $\psi$ is hyperbolic.

Definition 1.3. We say that a mapping $\varphi: B_{N} \rightarrow \mathbb{C}$ is semi-nonexpansive provided there exists a neighborhood $U_{w}$ of $w$ such that $|\varphi(z)-\varphi(w)| \leq|z-w|$ for all $z$ in $U_{w} \cap B_{N}$. 
The next section of the present paper shows that weighted composition operators with non-constant weight function and hyperbolic compositional symbol can be hypercyclic on $H\left(B_{N}\right)$. For some sources see [1]-[7].

\section{Main Result}

In this section we investigate the hypercyclicity of a hyperbolic weighted composition operator acting on $H\left(B_{N}\right)$.

Proposition 2.1. Let $\varphi$ be a nonzero holomorphic map on $B_{N}$ and $\psi$ be a hyperbolic map of $B_{N}$ with $w$ the Denjoy-Wolff point such that $\varphi(w) \neq 0$. If $\varphi$ is semi-nonexpansive, then $C_{\varphi, \psi}^{*}$ is not hypercyclic, but $C_{\varphi, \psi}$ is hypercyclic whenever $\varphi$ never vanishes on $B_{N}, C_{\psi}$ is hypercyclic and $|\varphi(w)|=1$.

Proof. Let $K$ be a compact subset of $B_{N}$. By Theorem 1.2, there exists a constant $c>0$ such that

$$
\left|1-<\psi_{n}(z), w>\right|^{2} \leq c\left(1-\left|\psi_{n}(z)\right|^{2}\right)
$$

for every $z \in K$ and every $n \in \mathbb{N}$. But $\left|1-<\psi_{n}(z), w>\right|^{2}=\left|w-\psi_{n}(z)\right|^{2}$, thus $\left|w-\psi_{n}(z)\right|^{2} \leq c\left(1-\left|\psi_{n}(z)\right|^{2}\right)$ for every $z \in K$ and every $n \in \mathbb{N}$. On the otherhand, since $\varphi$ is semi-nonexpansive, there exists a neighborhood $U_{w}$ of $w$ satisfying $|\varphi(w)-\varphi(z)| \leq|w-z|$ for every $z$ in $U_{w} \cap B_{N}$. Since $\psi_{n} \stackrel{k}{\rightarrow} w$, there exists $N$ such that for all $n>N, \psi_{n}(z) \in U_{w}$. Substituting $\psi_{n}(z)$ instead of $z$ in the previous relation we get

$$
\begin{aligned}
\left|\varphi(w)-\varphi\left(\psi_{n}(z)\right)\right| & \leq\left|w-\psi_{n}(z)\right| \\
& =\left|1-<\psi_{n}(z), w>\right| \\
& \leq c^{1 / 2}\left(1-\left|\psi_{n}(z)\right|^{2}\right)^{1 / 2},
\end{aligned}
$$

for every $n>N$. Now we apply the techniques used in [7]. Since $\psi$ is hyperbolic, thus $0<d(w)<1$ and by Theorem 1.2 we have

$$
\frac{|1-<\psi(z), w>|^{2}}{1-|\psi(z)|^{2}} \leq d(w) \frac{|1-<z, w>|^{2}}{1-|z|^{2}}
$$

for all $z \in B_{N}$. By substituting $\psi_{n}(z)$ for $\psi(z)$ in the above inequality, we get

$$
\frac{\left|1-<\psi_{n}(z), w>\right|^{2}}{1-\left|\psi_{n}(z)\right|^{2}} \leq d(w)^{n} \frac{|1-<z, w>|^{2}}{1-|z|^{2}}
$$


for every $z \in B_{N}$ and $n \in \mathbb{N}$. Also, note that since $K$ is compact, then there exists a constant $\beta>0$ such that $4 \frac{|1-<z, w>|^{2}}{1-|z|^{2}}<\beta$ for all $z$ in $K$. So it follows that

$$
\begin{aligned}
1-\left|\psi_{n}(z)\right|^{2} & =\left(1-\left|\psi_{n}(z)\right|\right)\left(1+\psi_{n}(z) \mid\right) \\
& \leq 2\left|1-<\psi_{n}(z), w>\right| \\
& \leq 4 \frac{\left|1-<\psi_{n}(z), w>\right|^{2}}{1-\left|\psi_{n}(z)\right|^{2}} \\
& \leq 4 \frac{|1-<z, w>|^{2}}{1-|z|^{2}} d(w)^{n} \\
& <\beta d(w)^{n} .
\end{aligned}
$$

Hence we obtain

$$
1-\left|\psi_{n}(z)\right|^{2} \leq \beta d(w)^{n}
$$

Now by using the relations $(*)$ and $(* *)$, we get

$$
\begin{aligned}
\mid 1-\frac{1}{\varphi(w)} \varphi\left(\psi_{n}(z) \mid\right. & <\frac{c^{\frac{1}{2}}}{|\varphi(w)|}\left(1-\left|\psi_{n}(z)^{2}\right|\right)^{1 / 2} \\
& \leq \frac{c^{\frac{1}{2}} \beta^{\frac{1}{2}}}{|\varphi(w)|} d(w)^{n / 2} .
\end{aligned}
$$

Since $0<d(w)<1$, thus $\sum_{n=0}^{\infty}\left|1-\frac{1}{\varphi(w)} \varphi\left(\psi_{n}(z)\right)\right|$ and so $\prod_{n=0}^{\infty} \frac{1}{\varphi(w)} \varphi\left(\psi_{n}(z)\right)$ converges uniformly on K. Define

$$
g(z)=\prod_{n=0}^{\infty} \frac{1}{\varphi(w)} \varphi\left(\psi_{n}(z)\right)
$$

Since $\varphi(w) \neq 0$ and $\psi_{n} \stackrel{k}{\rightarrow} w$, so there exists a neighborhood $U_{w}$ of $w$ such that $\varphi \circ \psi_{n} \neq 0$ on $U_{w}$ for all $n$ large enough. Let $z=\left(z_{1}, z^{\prime}\right) \in B_{N}$ where $z^{\prime}=$ $\left(z_{2}, \ldots, z_{N}\right) \in \mathbf{C}^{N-1}$. Define $f\left(z_{1}\right)=g\left(z_{1}, z^{\prime}\right)$, then $f$ is a nonzero holomorphic function. Thus $g$ is also a nonzero holomorphic function with respect to $z_{1}$. By the same method we can see that $g$ is holomorphic with respect to other variables $z_{2}, \ldots, z_{n}$. This implies that $g$ is a nonzero holomorphic function on $B_{N}$. Clearly, $C_{\varphi, \psi} g=\varphi(w) g$, and so $\varphi(w)$ is an eigenvalue of $C_{\varphi, \psi}$. But it is well-known that the adjoint of a hypercyclic operator has no eigenvector, thus $C_{\varphi, \psi}^{*}$ fails to be hypercyclic. Also, note that $C_{\varphi, \psi} M_{g}=M_{g}\left(\varphi(w) C_{\psi}\right)$, and $g$ 
has no zero in $B_{N}$ whenever $\varphi$ never vanishes. Thus, $M_{g}$ is one to one and has dense range and so $C_{\varphi, \psi}$ is quasisimilar to $\varphi(w) C_{\psi}$. Now if $|\varphi(w)|=1$ and $C_{\psi}$ is hypercyclic, then $\varphi(w) C_{\psi}$ and so $C_{\varphi, \psi}$ is also hypercyclic on $H\left(B_{N}\right)$. This completes the proof.

\section{References}

[1] P. S. Bourdon and J. H. Shapiro, Cyclic Phenomena for composition operators, Mem. Amer. Math. Soc., 596 (1997).

[2] K. C. Chan and J. H. Shapiro, The cyclic behavior of translation operators on Hilbert spaces of entire functions, Indiana Univ. Math. J., 40 (1991), 1421-1449.

[3] C. C. Cowen and B. D. MacClur, Composition Operators on Spaces of Analytic Functions, CRC Press, Boca Raton (1995).

[4] R. M. Gethner and J. H. Shapiro, Universal vectors for operators on spaces of holomorphic functions, Proc. Amer. Math. Soc., 100 (1987), 281-288.

[5] G. Godefroy and J. H. Shapiro, Operators with dense invariant cyclic vector manifolds, J. Func. Anal., 98 (1991), 229-269.

[6] J. H. Shapiro, Composition Operators and Classical Function Theory, Springer-Verlag, New York (1993).

[7] B. Yousefi and H. Rezaei, Hypercyclic property of weighted composition operators, Proc. Amer. Math. Soc., 135, No. 10 (2007), 3263-3271. 
